\title{
Recent update of the 2017 Korean Association for the Study of the Liver (KASL) treatment guidelines of chronic hepatitis C: Comparison of guidelines from other continents, 2017 AASLD/IDSA and $2016 \mathrm{EASL}$
}

\author{
Jong Eun Yeon \\ Department of Internal Medicine, Korea University College of Medicine, Seoul, Korea
}

The paradigm for the treatment of chronic hepatitis $\mathrm{C}(\mathrm{CHC})$ has been changed due to the development of direct acting antivirals (DAAs) of hepatitis $\mathrm{C}$ virus (HCV). The high sustained virologic response rate and ease of administration makes the DAAs approach ideal to contribute to the complete eradication of HCV. Currently, treatment options for individual patients vary depending on the genotype or subtype of $\mathrm{HCV}$, presence or absence of liver cirrhosis, previous experience of antiviral treatment or resistance associated substitutions. Because of drug avalilability, cost-effectiveness, preference, compliance and greater possibility of desirable effects and presumed patient-important outcomes may vary between countries, treatment options for individual patients are different. The review focuses on the comparing the current treatment options for CHC in other continents with the 2017 Korea Association for the Study of the Liver guidelines. (Clin Mol Hepatol 2018;24:278-293)

Keywords: Hepatitis C virus; Direct acting antiviral; Resistance associated substitutions; Genotype; Liver cirrhosis

\section{INTRODUCTION}

Since the development of direct acting antivirals (DAA) against hepatitis $\mathrm{C}$ virus $(\mathrm{HCV})$, the paradigm for the pharmacological management of chronic hepatitis $C$ has been changed. The ease of administration and high sustained virologic response rate (SVR) makes

\footnotetext{
Abbreviations:

2017 KASL guideline, 2017 Korean Association for the Study of the Liver (KASL) guideline on 'hepatitis C': treatment of chronic hepatitis $C_{;}$ALT, alanine aminotransferase; anti-HCV, antibody to hepatitis $C_{;}$AST, aspartate aminotransferase; ASV, asunaprevir; /r, /ritonavir; CC, compensated liver cirrhosis; CTP, Child-Pugh Turcotte; $C H C$, chronic hepatitis C; CKD, chronic kidney disease; eGFR, estimated glomerular filtration rate; DAA, direct-acting antivirals; DCV, daclatasvir; DC, decompensated liver cirrhosis; EBR/GZR, elbasvir/grazoprevir; ESRD, end-stage renal disease; GFR, glomerular filtration rate; G/P, glecaprevir/ pibrentasvir; HBV, hepatitis B virus; HCC, hepatocellular carcinoma; HCV, hepatitis C virus; HIV, human immunodeficiency virus; LC, liver cirrhosis; LED/ SOF, ledipasvir/sofosbuvir; LT, liver transplantation; MELD, model for end-stage liver disease; NS, nonstructural protein; NS3, nonstructural protein 3; NS5A, nonstructural protein 5A; Opr+D, ombitasvir/paritaprevir/ritonavir and dasabuvir; $P C R$, polymerase chain reaction; PegIFN, peginterferon alpha; PI, protease inhibitor; PR, PEG-interferon alpha+ribavirin therapy; $R^{*}$, ribavirin started from $600 \mathrm{mg} / \mathrm{d}$; R, weight-based ribavirin; RASs, resistance-associated substitutions; SIM, simeprevir; SOF, sofosbuvir; SOF/VEL, sofosbuvir/velpatasvir; SOF/VEL/VOX sofosbuvir/velpatasvir/voxilaprevir; SVR, sustained virologic response rate; TN, treatment-naïve; TE, treatment-experienced; wk, weeks
}

Corresponding author : Jong Eun Yeon

Department of Internal Medicine, Korea University College of Medicine, 148 Gurodong-ro, Guro-gu, Seoul 08308, Korea Tel: +82-2-2626-1030, Fax: +82-2-2626-1038

E-mail: jeyyeon@hotmail.com

https://orcid.org/0000-0002-0510-7371 
the DAA approach ideal to contribute to the complete eradication of $\mathrm{HCV}$.

Currently, treatment options for an individual patient vary depending on the genotype (GT), subtype, previous treatment experience, presence or absence of liver cirrhosis (LC) or resistance associated substitutions (RASs). Also, re-treatment options for the patients who failed previous DAA therapy are limited. Since the development of the first generation DAA, there has been much progress, including the introduction of pan-genotypic new DAA, DAA which have activity against HCV with RASs, and the publication of many novel research results from both Korea and other countries.

In Korea, the guidelines regarding "chronic hepatitis C (CHC)" were first developed in 2004 and revised in 2013', 2015', and $2017^{3}$ by the Korea Association for the Study of the Liver (KASL). The HCV guidance by the American Association for the Study of Liver Disease (AASLD) and the Infectious Disease Society of America (IDSA) was recently released in September 2017. ${ }^{4}$ The European Association for the Study of the Liver (EASL) recommendation on treatment of hepatitis C $2016^{5}$ is expected to be revised soon. Because drug availability, greater possibility of desirable effects, cost-effectiveness, preference, and compliance and presumed patient-important outcomes may vary between countries, treatment options for individual patients are different. In this article, I intended to compare the treatment options for $\mathrm{CHC}$ in other continents with the $2017 \mathrm{KASL}$ guidelines.

\section{CURRENTLY AVAILABLE DAAs IN KOREA, YEAR 2017}

In Korea, the currently available DAA are ledipasvir/sofosbuvir (LED/SOF), sofosbuvir (SOF), daclatavir (DCV), asunaprevir (ASV), ombitasvir/paritaprevir/ritonavir (OPr), dasabuvir(D) and elbasvir/ grazoprevir (EBR/GZR). Sofosbuvir/velpatasvir (SOF/VEL), Glecaprevir/pibrentasvir (G/P) and sofosbuvir/velpatasvir/voxilaprevir (SOF/ VEL/VOX) are not available yet. Simeprevir (SIM) is not available in Korea.

In Korea, the criteria for payment under the medical care benefits are as follows: for GT1b patients with treatment-naive (TN) and treatment-experienced (TE) CHC and compensated cirrhosis (CC), OPr+D, EBR/GZR (add R if protease inhibitor [PI]+PR fail), and DCV+ ASV; for GT1b patients who are RAS positive or for those who are not qualified for DCV+ASV (including decompensated LC (DC), post-liver transplantation [LT], or side effects), LED/SOF or DCV+SOF; for GT1a patients, LED/SOF, OPr+D+ribavirin (R), EBR/GZR ( $\pm R$ ), $\mathrm{DCV}+\mathrm{SOF}$, SOF+PR; for GT1 post LT patients with fibrosis less than
F2, OPrD+R; for DC or post-LT GT1 patients, LED/SOF+R or DCV+ $S O F+R$; for GT2 patients, SOF+R; for GT3 patients, $D C V+S O F( \pm R)$; for GT4 patients, OPr+R, EBR/GZR( $\pm R)$, SOR+PR or SOF+R. Treatment duration and combination with or without ribavirin may differ from individual patients with each GT. Besides DAA, it is possible to prescribe PEG-interferon alpha. Currently available DAA and the drugs covered for payment under the medical care benefits for each GT are listed in Table 1.

\section{NEW DAAs, YEAR 2017}

Differently from the 2015 KASL guidelines, which introduced DAA including LED/SOF, SOF, DCV, ASV, OPr+D, SOF+PR and SIM, the 2017 KASL guidelines included new DAA, such as EBR/GZR, SOF/VEL, G/P and SOF/VEL/VOX. The results from clinical studies for new DAA will be described briefly.

\section{EBR/GZR}

According to the $2017 \mathrm{KASL}$ guidelines, EBR/GZR is recommended for GT1 and GT4 patients, respectively. In a phase 3 study of TN CHC patients including GT1b, GT1a, GT4, and GT6 treated with EBR/ GZR showed an SVR of $99 \%, 92 \%, 100 \%$, and $80 \%$, respectively. NS5A RASs were detected in 12\% of GT1a patients, and the SVR was significantly lower in patients with RASs compared to those without RASs (58\% vs. 99\%). A pooled analysis of phase 2 and 3 clinical trials revealed that the SVR was $100 \%$ in GT1a infected patients with baseline NS5A RASs treated with EBR/GZR and ribavirin for 16 or $18 w k$.

\section{$\mathrm{G} / \mathrm{P}$}

A phase 3 study that assessed the efficacy and safety of 8- or 12wk G/P treatment in HCV GT1 patients without LC ( $n=703$, IFN-based TE patients 28\%, SOF-based TE patients $0.4 \%$ ) showed an SVR of $99 \%$ and $99.7 \%$, respectively. ${ }^{8}$ A pooled analysis of phase 2 or 3 study in patients with GT1-6 chronic HCV infection without cirrhosis (interferon-based TE patients 23\%, SOF-based TE patients 1\%) treated with G/P for 8 or 12 wk, showed the following SVR in 8 vs. 12 wk: GT1 (100\% vs. $100 \%)$, GT2 (99\% vs. $100 \%)$, GT3 (97\% vs. $98 \%)$, GT4 $(100 \%$ vs. $100 \%)$, GT5 (100\% vs. $100 \%)$, and GT6 $(100 \%$ vs. $100 \%){ }^{9}$ A phase 3 study among HCV GT1, 2, 4, 5, 6 infected patients with CC ( $n=146$, IFN-based TE 17\%, SOF-based TE 8\%) treated with G/P for 12 wk showed SVR of $99 \%, 100 \%, 100 \%, 100 \%$, 
Table 1. Currently available DAAs and drugs covered for payment under the medical care benefits in Korea

\begin{tabular}{|c|c|c|c|}
\hline Direct acting antivirals (DAA) & KMFD approved* & $\begin{array}{l}\text { Drugs covered for payment } \\
\text { under the medical care } \\
\text { benefit** }\end{array}$ & 2017 KASL guideline $e^{* * *}$ \\
\hline Ledipasvir/sofosbuvir (LED/SOF) & Yes & $\begin{array}{l}\text { GT1a, GT1b with RAS, } \\
\text { decompensated LC, post-LT }\end{array}$ & GT1b, GT1a, GT4, GT5, GT6 \\
\hline Sofosbuvir (SOF) & Yes & $\begin{array}{l}\text { Comined with ribavirin, } \mathrm{PR} \text { or } \\
\text { DCV }\end{array}$ & Refer to below \\
\hline Daclatasvir (DCV) & Yes & $\begin{array}{l}\text { DCV+ASV in GT1b, DCV+SOF in } \\
\text { GT3, DCV+SOF in GT1a, GT1b } \\
\text { with RAS, GT1 with decomp. } \\
\text { LC, post LT }\end{array}$ & $\begin{array}{l}\text { DCV+ASV in GT1b, DCV+SOF in } \\
\text { GT1-GT6, DCV+SOF in } \\
\text { decompensated LC \& post-LT }\end{array}$ \\
\hline Aunaprevir (ASV) & Yes & DCV+ASV in GT1b & $\mathrm{DCV}+\mathrm{ASV}$ in GT1b \\
\hline Ombitasvir/paritaprevir/ritonavir (OPr) & Yes & OPrD in GT1, OPr in GT4 ${ }^{\dagger}$ & OPrD in GT1, OPr in GT4 ${ }^{\dagger}$ \\
\hline Dasabuvir (D) & Yes & OPrD in GT1, OPr in GT4 & OPrD in GT1, OPr in GT4 ${ }^{\dagger}$ \\
\hline Elbasvir/grazoprevir (EBR/GZP) & Yes & GT1a, GT1b, GT4 ${ }^{\dagger}$ & GT1a, GT1b, GT4 \\
\hline Glecaprevir/pibrentasvir (G/P) & $\begin{array}{l}\text { Expected to be } \\
\text { approved }\end{array}$ & Not yet & $\mathrm{GT1}-6^{\ddagger}$ \\
\hline Sofosbuvir/velpatasvir (SOF/VEL) & No & None & $\mathrm{GT1}-6^{\ddagger}$ \\
\hline \multirow[t]{2}{*}{ Sofosbuvir/velpatasvir/voxilaprevir (SOF/VEL/VOX) } & \multirow[t]{2}{*}{ No } & \multirow[t]{2}{*}{ None } & GT1-6 with DAA failure ${ }^{t \dagger}$ \\
\hline & & & TN \& TE GT3 with LC \\
\hline Peg-interferon with ribavirin (PR) & Yes & GT1-3 & $\begin{array}{l}\text { GT2, 3, 5, 6, for whom DAA is } \\
\text { not indicated }\end{array}$ \\
\hline Sof+ribavirin & Yes & $\mathrm{GT} 2,4$ & GT2 \\
\hline Sof $+P R$ & Yes & GT1, GT4 & Not recommended \\
\hline
\end{tabular}

KMFD, Korean Ministry of Food and Drug Safety; HIRA, Health Insurance Review and Assessment service.

*Approved state in DEC. 2017, may change during the publication, please refer to website (www.mfds.go.kr) for further information; **Refundable by medical insurance, may change during the publication, please refer to website (www.hira.or.kr) for further information; ${ }^{* *}$ Indicated in 2017 KASL HCV guideline, may differ from medicare covered drug, please refer to website (www.kasl.org) for further information including ribavirin combination and treatment duration; ${ }^{\dagger}$ May differ from each patient with presence or absence of treatment experience, liver cirrhosis or RAS in terms of adding ribavirin, treatment duration; ${ }^{\ddagger}$ Treatment duration may differ from each genotype, presence or absence of treatment experinece or cirrhosis, please refer to website (www.kasl.org) for further information include ribavirin combination and treatment duration; ${ }^{\dagger \dagger}$ Treatment indication may differ from each types of DAA failure, please refer to website (www.kasl.org) for further information include ribavirin combination and treatment duration.

$100 \%$, respectively. ${ }^{10}$ In a phase 2 study regarding G/P treatment for 12 or 16 wk in GT3 patients without $L C$ and G/P treatment for 16 wk in TE GT3 patients with LC (PR experienced patients 54\%, SOF+R \pm PEG-IFN experienced patients $46 \%$ ), SVR were $91 \%$, 96\%, $96 \%$, respectively. ${ }^{11}$ The results of clinical trials for $\mathrm{G} / \mathrm{P}$ in patients who failed DAA treatment will be described in each section.

\section{SOF/VEL}

In a phase 3, double-blind, placebo-controlled study among untreated and previously treated HCV GT1b, GT1a, GT2, GT4, GT5, or GT6 infected patients (LC 19\%, TE 32\%), the SVR of SOF/VEL treatment for 12 wk were $99 \%, 98 \%, 100 \%, 100 \%, 97 \%$, and $100 \%$, respectively. ${ }^{12}$ In a phase 3 study comparing a $12 \mathrm{wk}$ SOF/VEL therapy to a 12wk SOF+R therapy in GT3 patients (LC 29\%, TE 26\%), the SVR were $95 \%$ and $80 \%$, respectively. The prevalence of RAS in GT3 patients treated with SOF/VEL was 16\%. In patients with or without NS5A RAS, the SVR were $88 \%$ and $97 \%$, respectively. ${ }^{13}$ In TN GT3 patients treated with SOF/VEL, SVR noncirrhotic and cirrhotic patients were $98 \%$ and $93 \%$, respectively. In TE patients, SOF/VEL treatment for $12 \mathrm{wk}$ in noncirrhotic and cirrhotic patients resulted in a SVR of $91 \%$ and $89 \%$, respectively. ${ }^{13}$

\section{SOF/VEL/VOX}

In a phase 3, open-label trial, HCV infected GT1-6 patients, who 
had not been previously treated with DAA, were assigned randomly to groups administered SOF/VEL/VOX for 8 wk or SOF/VEL for 12 wk ( $n=941$, LC 18\%, IFN-TE patients 23\%, except GT3 LC patients) and showed SVR of 95\% and $98 \%$, respectively. The SVR of SOF/VEL/VOX treatment for 8 wk compared to SOF/VEL treatment for 12 wk in each GT was as follows: GT1b (97\% vs. 97\%), GT1a (92\% vs. $99 \%)$, GT2 (97\% vs. $100 \%)$, GT3 ( $99 \%$ vs. $97 \%)$, GT4 ( $94 \%$ vs. 98\%), GT5 (all patients assigned to receive SOF/VEL/VOX, 94\%), GT6 (100\% vs. 100\%). In patients without or with LC, the SVR for each treatment group were $96 \%$ vs. $98 \%$, and $91 \%$ vs. $99 \%$, respectively. The prevalence of baseline NS3 or NS5A RASs was $50 \%$ in each group. In patients with baseline RAS, the SVR was $94 \%$ and $99 \%$ in the SOF/VEL/VOX and SOF/VEL groups, respectively. In a phase 3 study that compared SOF/VEL/VOX therapy for $8 \mathrm{wks}$ with SOF/VEL therapy for 12 wks in GT3 patients with LC (TE 31\%), the SVR were $96 \%$ and $96 \%$, respectively. ${ }^{14}$ The results of clinical trials of SOF/VEL/VOX in patients who failed treatment with DAA will be described in each section.

\section{DEFINITION, SPECIAL SITUATION IN KOREA}

In Korea, the number of CHC patients who have been treated with first generation DAA, such as boceprevir or telaprevir is very limited. As a result, "treatment experienced (TE)" refers to patients with interferon (or PEG-IFN) with or without ribavirin therapy, unless otherwise mentioned. According to the 2017 KASL guidelines, ${ }^{3}$ GT1 patients who cannot be sub-typed should be treated as if they were infected with GT1a. Decompensated liver cirrhosis (DC) refers to patients who have Child-Pugh-Turcotte class of more than B or experienced decompensated events. SVR refers to SVR at $12 \mathrm{wk}$ after the end of treatment unless otherwise mentioned.

\section{RAS DETECTION AND INDICATION FOR PRE- TREATMENT ASSESSMENT}

Standardized detection of NS5A or drug-specific RAS is not available. However, sequencing of $L 31$ and $Y 93$ mutations in the NS5A region for GT1b patients scheduled for treatment with DCV+ASV was recently approved. For GT1a patients who are scheduled for treatment with EBR/GZR, the RAS test is recommended although it is not approved yet. Patients with "undetermined" RAS test are treated similarly with RASs positive patients. The RAS test for GT3 or GT4 patients is not available in Korea yet.

\section{QUALITY OF EVIDENCE AND STRENGTH OF RECOMMENDATION IN 2017 KASL GUIDELINE}

The quality of evidence was classified according to the GRADE (Grading of Recommendations, Assessment, Development, and Evaluation) system. ${ }^{15}$ The level of evidence was defined as follows; A, the highest level of evidence with the smallest possibility of changes in the conclusion; B, a moderated level of potential changes; and C, the lowest level of evidence with the greatest possibility of changes. The strength of a recommendation was also classified according to the GRADE system. Each study was classified as strong recommendation (1) or weak recommendation (2) based on the quality of evidence, the balance between the desirable and undesirable effect of an intervention, and socioeconomic aspects including cost or availability.

\section{TREATMENT APPROACH TO PATIENTS WITH CHRONIC HEPATITIS C (CHC) AND COMPEN- SATED LIVER CIRRHOSIS (CC)}

The recent updates of the 2017 KASL guidelines regarding treatment for each genotype are summarized in Table 2. The 2017 HCV guidance of AASLD/IDSA are summarized in Table 3. The recommendations of the EASL regarding treatment of hepatitis $C$ 2016 are summarized in Table 4. Different treatment options for the same genotypes from other societies will be discussed.

\section{Treatment of treatment-naïve (TN) and treatment- experienced (TE) GT1b patients with chronic hepatitis (CHC) and compensated cirrhosis (CC)}

The following seven regimens are recommended for the treatment of GT1b patients: LED/SOF regimen for 12 wk (shorter treatment duration to $8 \mathrm{wk}$ may be considered in TN non-cirrhotic patients with HCV RNA less than 6 million IU/mL and without human immunodeficiency virus [HIV]-coinfection) in TN CHC/CC or TE CHC and $12 w k+R$ or 24 wk for TE patients with CC, EBR/GZR for 12 $w k, O P r+D$ for $12 w k, D C V+S O F$ for $12 w k$ for patients without LC and 12 wk+R or 24 wk for patients with LC or DCV+ASV for 24 wk are currently recommended. Although it is not approved in Korea yet, G/P treatment for 8 wk for patients without LC and 12 wk for patients with LC, or SOF/VEL for 12 wk is the one of the other treatment options.

Differently from AASLD and EASL guidelines, DCV+ASV for 24 


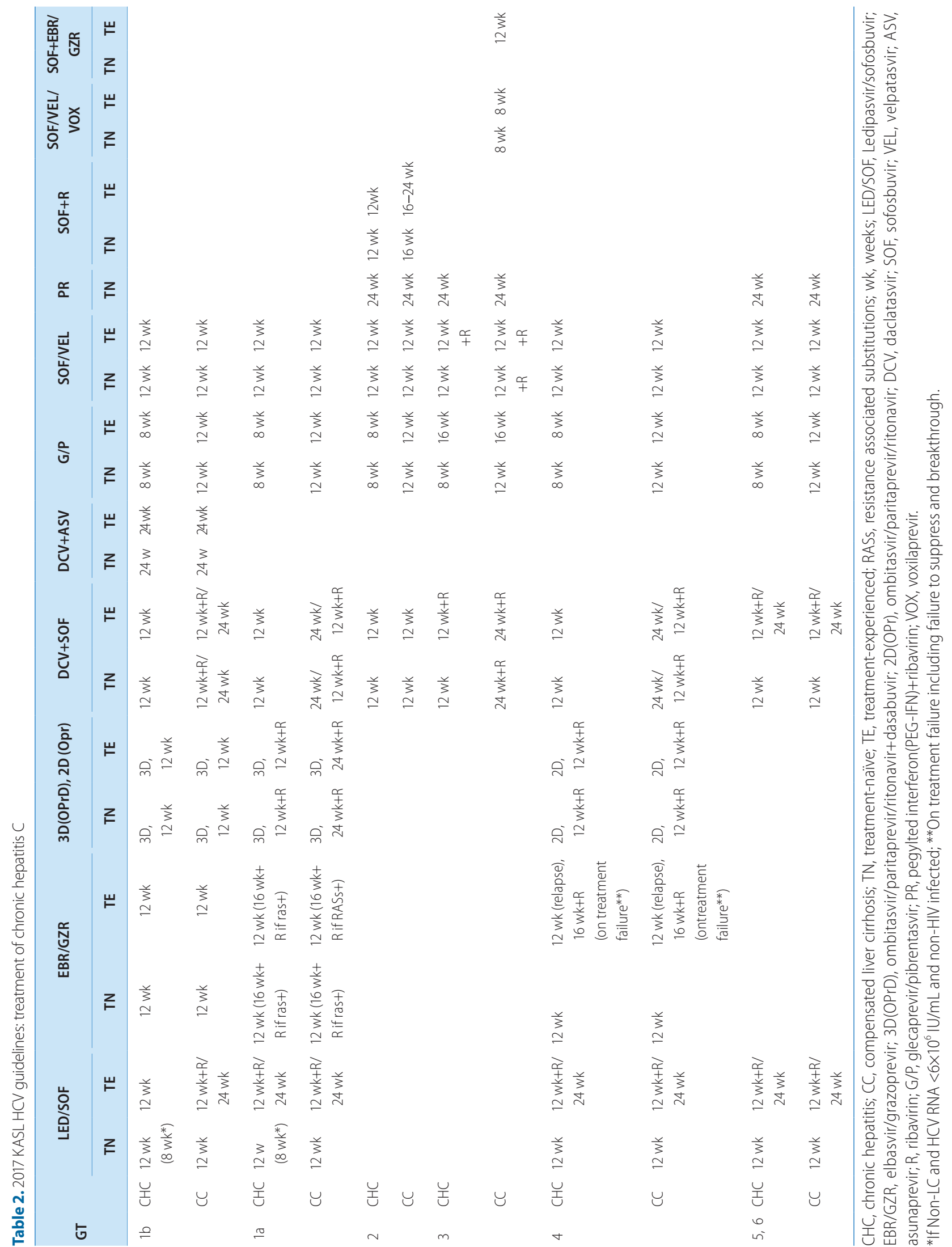


Jong Eun Yeon

Comparison of $\mathrm{HCV}$ treatment guidelines in different societies

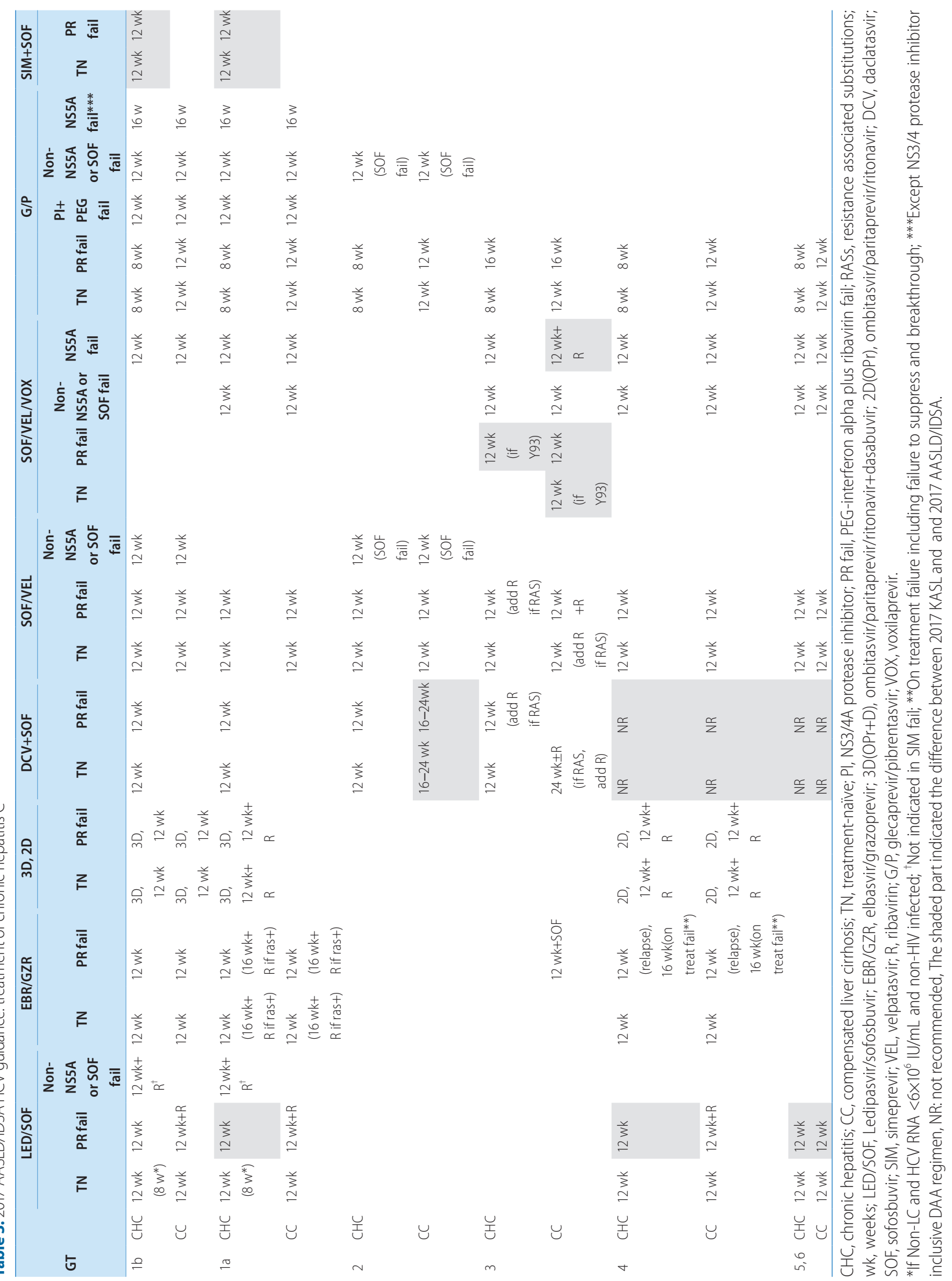


Volume 24 Number 3 September 2018

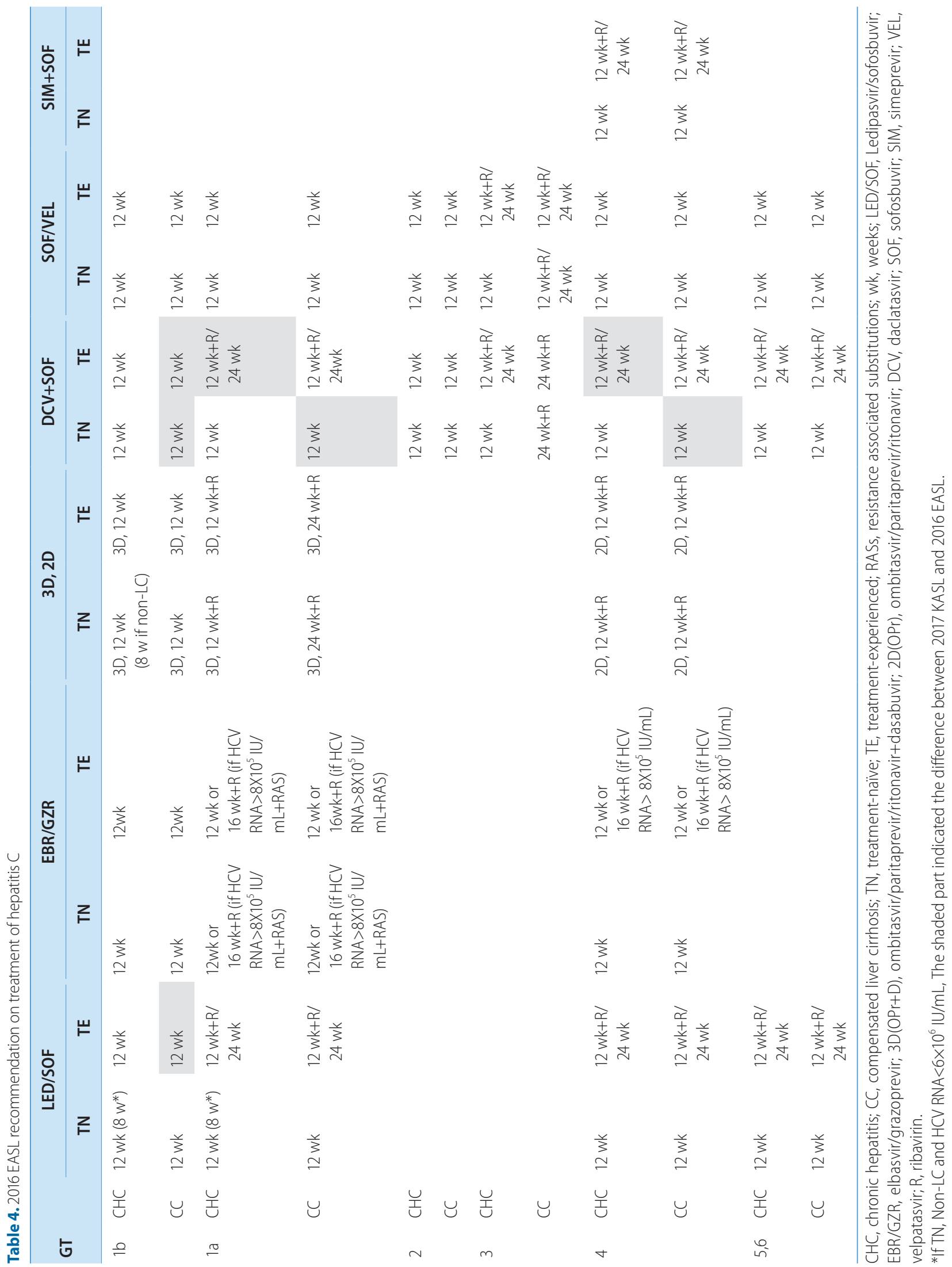


wk is one of treatment options for GT1b patients in Korea (level of evidence and strength of recommendation, A2). A post hoc analysis of a phase 3 clinical study regarding treatment of Asian genotype $1 \mathrm{~b}$ patients ( $\mathrm{n}=747$, including 78 Korean, LC 32\%) for $24 \mathrm{wk}$ with ASV plus DCV revealed a SVR of $92 \%, 79 \%, 80 \%$ in the TN, IFN ineligible, IFN-non-response group, respectively. ${ }^{16}$ The presence of baseline NS5A RASS (L31 or Y93) significantly reduced SVR. A pooled data analysis from five clinical studies ( $n=979$, TN $30 \%$, LC 22\%) demonstrated a SVR of $39 \%$ in patients with NS5A RASs compared to a SVR of $94 \%$ in patients without RASs. ${ }^{17}$ In this study, the prevalence of NS5A RAS was 13-14\%.

Different from the 2015 KASL guidelines, a shorter treatment for 8 wk with LED/SOF can be considered in TN non-cirrhosis GT1b patients with HCV RNA less than 6 million IU/mL and without HIV infection. In a real-life observational cohort study ${ }^{18}$, patients who completed 8 wk of LED/SOF treatment had a SVR of $93 \%$, whereas those who completed 12 wk of treatment had a SVR of $97 \%$. In another real-life study, the SVR of TN, GT1b patients without cirrhosis treated for 8 wk with LED/SOF was $99 \%{ }^{19}$ In addition, meta-analysis of six real world cohorts comprising of 5,637 patients showed that the relapse rate was comparable between 8-and 12-wk LED/ SOF treatments (relative risk, $0.99,95 \% \mathrm{Cl} 0.98-1.00$ ). ${ }^{19}$ Based on these data, the 2017 KASL guidelines adopted a shorter LED/SOF treatment for 8 wk in TN non-cirrhotic patients with HCV RNA less than 6 million IU/mL and without HIV infection.

Different from the 2016 EASL guidelines, the treatment duration of the $D C V+S O F$ regimen in $L C$ patients is $12 w k+R$ or $24 \mathrm{wk}$. In a cohort study that recruited 768 genotype 1 infected patients (GT1b $46 \%$, LC $73 \%$, TN $16 \%){ }^{20}$ SVR was assessed according to treatment duration (12 wk vs. 24 wk DCV+SOF with or without ribavirin). In patients with cirrhosis, treated with DCV+SOF for $12 \mathrm{wk}, 12$ $w k+R$, $24 w k$, or $24 w k+R$, SVR were 87\% (82/94), 92\% (23/25), $94 \%$ (323/343), $98 \%(100 / 102)$, respectively $(P=0.0152)$. This study suggested that cirrhosis status and treatment experience influenced SVR. Based on these data, the 2017 KASL guidelines recommended a $12 \mathrm{wk}+\mathrm{R}$ or $24 \mathrm{wk}$ DCV+SOF treatment in patients with liver cirrhosis. The 2016 EASL guidelines recommended a 12 wk DCV+SOF treatment in GT1b patients regardless of treatment experience or the presence of cirrhosis.

In AASLD/IDSA HCV guidance, SIM combined with SOF for 12 wk is recommended in GT1b TN or TE CHC.

\section{Treatment of TN and TE GT1a patients with $\mathrm{CHC}$ and CC}

The following six regimens are recommended with comparable efficacy for the treatment of GT1a patients: LED/SOF treatment for $12 \mathrm{wk}$ (shorter treatment duration to 8 wk may be considered in TN non-cirrhotic patients with HCV RNA less than 6 million $\mathrm{IU} / \mathrm{mL}$ and non-HIV infected) in TN patients and $12 \mathrm{wk}+\mathrm{R}$ or $24 \mathrm{wk}$ for TE patients, EBR/GZR for 12 wk (if RAS+, 16 wk+R), OPr+D+R for 12 wk for patients without cirrhosis and 24 wk for patients with cirrhosis, DCV+SOF for $12 \mathrm{wk}$ for patients without cirrhosis and $24 \mathrm{wk}$ or $12 \mathrm{wk}+\mathrm{R}$ for patients with cirrhosis. Although it is not approved in Korea yet, G/P treatment for 8 wk for patients without cirrhosis and 12 wk for patients with cirrhosis, or SOF/VEL for 12 wk is the one of the other treatment options.

Different from the AASLD guidelines, LED/SOF $12 w k+R$ or $24 w k$ is recommended for TE GT1a chronic hepatitis patients without cirrhosis in 2017 KASL guidelines. A previous study ${ }^{21}$ regarding TE patients ( $n=440$, GT1a 79\%, LC 20\%), under LED/SOF treatment for 12 wk, $12 w k+R$, $24 w k$, or $24 w k+R$ showed no major differences in SVR; 94\% (102/109), 96\% (107/111), 99\% (108/109), 99\% (110/111) in each group, respectively. In patients with cirrhosis, SVR was 86\% (19/22), $82 \%$ (18/22), 100\% (22/22), 100\% (22/22) respectively. Accordingly, AASLD recommended LED/SOF treatment for 12 wk without ribavirin for chronic hepatitis and 12 wk with ribavirin for $L C$ patients. In GT1a patients, the presence of baseline NS5A or LED specific RASs significantly reduced SVR. In TE GT1a patients, SVR of LED/SOF was $76 \%$ (22/29) in patients with LED-specific RAS, which is lower than the $97 \%$ (409/420) observed in patients without LED-specific RAS. ${ }^{22}$ In LC patients, SVR was 77\% (10/13) and 96\% (216/224) in patients with or without LED-specific RAS, respectively. Even in patients without cirrhosis, SVR was 75\% (12/16) and 98\% (193/196) in patients with or without LED-specific RAS. The prevalence of LED-specific RAS and NS5A RAS was reported up to $8.3 \%$ and $13.0 \%$, respectively. ${ }^{22}$ In addition, the RAS test for GT1a is not available in Korea. Accordingly, the 2017 KASL guidelines suggested the addition of ribavirin to 12 wk LED/SOF therapy or the extension of LED/SOF therapy to 24 wk in TE GT1a patients with or without cirrhosis.

As described previously, the 2017 KASL guidelines recommend a $12 w k+R$ or $24 w k$ DCV+SOF treatment in GT1a patients with liver cirrhosis. The 2016 EASL guidelines recommended a 12 wk treatment with DCV+SOF in TN GT1a patients with cirrhosis and a $12 \mathrm{wk}$ $+R / 24$ wk in TE patients without cirrhosis. SIM combined with SOF for 12 wk is recommended in TN and TE GT1a non-cirrhotic patients in AASLD/IDSA HCV guidance.

\section{Treatment of TN and TE GT2 patients with $\mathrm{CHC}$ and CC}

The following five regimens are available for GT2 patients: SOF+R 
12 wk for TN \& TE patients with CHC, 16wk for TN LC patients and 16-24 wk for TE LC patients or DCV+SOF 12 wk. G/P treatment for 8 wk for patients with CHC and 12 wk for patients with CC or SOF/VEL for $12 \mathrm{wk}$ is one of the other treatment options although they are not approved in Korea yet. PR 24 wk for TN patients may be considered if there is no available DAA treatment option (e.g. chronic kidney disease, CKD).

Different from the treatment guidelines from other continents, $\mathrm{SOF}+\mathrm{R}$ is the only regimen covered for payment under the medical care benefits for GT2 patients in Korea.

Previous studies from western countries have shown that the SVR of SOF+R treatment for 12 wk in TN GT2 patients with CHC was 96$98 \%{ }^{23,24}$ In patients with cirrhosis, studies for the optimal duration of SOF+R are very limited. SVR of $12 w k, 16 w k$ or 20 wk treatment with SOF+R in LC were 72-100\%, 95-100\% and 91\% although that was not a direct comparison. ${ }^{25,26}$ In a real-life study in Europe (TE 30.5\%, LC 26.8\%, DC 11\%), ${ }^{25}$ the overall SVR of $12 \mathrm{wk} / 16 \mathrm{wk}$ $\mathrm{SOF}+\mathrm{R}$ treatment was $88.2 \%$. In non-cirrhotic patient, SVR of $12 \mathrm{wk}$ or 16 wk treatment is $91 \%$ and $92.9 \%$. In patients with $\mathrm{LC}$ treated of 12 or 16 wk, SVR was $79.0 \%$ and $83 \%$. In another real-life study (TE $43 \%$, LC 58\%), ${ }^{26} 12$ wk treatment with SOF+R in patients with advanced fibrosis $(n=123)$ or $16 \mathrm{wk} / 20 \mathrm{wk}$ treatment in patients with LC $(n=168)$ showed SVR of $95 \%$. In patients with LC treated for 16 wk or 20 wk, SVR was 95\% (86/91) and 91\% (75/82).

In Korea, the SVR of 12-wk SOF+R treatment was 98\% (177/181) in TN and 97\% (32/33) in TE patients without liver cirrhosis. In patients with CC, the SVR of 16-week SOF+R treatment was 96\% (50/ 52), although it is not possible to distinguish between TN and TE patients. $^{27}$

Regarding DCV+SOF, the 2017 KASL guidelines recommended a 12 wk treatment for both TN and TE, non-LC and $L C$ patients which is different from the AASLD guidelines, which recommended a 12 wk therapy for non-LC and 16-24 wk therapy for LC patients. There are only a few studies regarding DCV and SOF therapies in patients with GT2. In phase 2 studies of 24 wk DCV+SOF treatment, the SVR was $92 \%$ (24/26) in TN patients. ${ }^{28}$ Based on sub-group analysis of genotype 2 patients in a real-life study (GT1-4, $n=2,612$, TE 53\%), DCV+SOF therapy for 12 wk showed a 100\% SVR for all 17 patients without cirrhosis and all 29 patients with liver cirrhosis, although it is not possible to distinguish TE from TN patients. ${ }^{29}$

\section{Treatment of TN and TE GT3 patients with $\mathrm{CHC}$ and CC}

The following five regimens are available for GT3 patients: $\mathrm{DCV}+\mathrm{SOF}$ for $12 \mathrm{wk}$ for TN CHC and $12 w k+\mathrm{R}$ for TE CHC, and
24wk+R for TN \& TE patients with cirrhosis. Although it is not approved yet, G/P treatment for 8wk for TN CHC patients, 12wk for TN LC patients, 16wk for TE CHC and LC patients or SOF/VEL for 12wk for TN CHC and 12wk+R for TN LC, TE CHC and TE LC patients or SOF/VEL/VOX for 8wk for TN or TE LC patients is one of the other treatment options. PR treatment for 24 wk in TN patients may be considered if there is no available DAA treatment option (i.e. CKD). EBR/GZR+SOF treatment for $12 w k$ can be considered TE GT3 patients with cirrhosis.

$\mathrm{DCV}+\mathrm{SOF}$ is the only regimen covered for payment under the medical care benefits for GT3 patients in Korea. In a phase 3 study of DCV+SOF therapy for 12 wk (TE 33\%, LC 21\%), the SVR for TN and TE patients was 90\% (91/101) and 86\% (44/51), respectively. In TN patients without or with LC, SVR was $97 \%$ and $58 \%$, respectively. ${ }^{30}$ In TE patients without or with liver cirrhosis, SVR was $94 \%$ and $69 \%$, respectively. In patients infected with HCV variants with RASs, SVR of DCV+SOF therapy was $83 \%$ in non-LC and $30 \%$ in LC patients. ${ }^{30}$ Based on sub-group analysis of genotype 3 patients in a real-life study (GT1-4, $n=2,612, T E 53 \%), D C V+S O F$ therapy for 12 wk showed a SVR of $95 \%$ (18/19) in non-LC patients, DCV+SOF+R for 12 wk showed a SVR of 92\% (121/131) in patients with liver cirrhosis although it is not possible to distinguish between the TN and TE patients. ${ }^{29}$ In a real life study regarding DCV+SOF therapy for 12 or 24 wk (TE 47\%, DC 20\%, ribavirin combination $86 \%$ and $78 \%$ ) in patients with LC, the SVR was $92 \%(34 / 37)$ and 95\% (89/94), respectively. The overall SVR in TN and TE patients was $93 \%$ and $94 \%$, respectively. ${ }^{31}$ In Korea, the RAS test for GT3 patients is not available. Based on these results and the challenges to treat GT3 LC patients, the 2017 KASL guidelines recommended a $12 \mathrm{wk}$ DCV+SOF therapy for TN CHC patients, a $12 \mathrm{wk}+\mathrm{R}$ therapy for TE CHC patients and a $24 \mathrm{wk}+\mathrm{R}$ therapy for TN- and TE-LC patients.

In a phase 3 study comparing SOF/VEL treatment for $12 \mathrm{wk}$ and SOF+R for $12 w k$, SVR were as follows: TN CHC (98\% vs. 90\%), TN LC (93\% vs. $73 \%)$, TE CHC (91\% vs. $71 \%$ ) and TE LC (89\% vs. $58 \%)^{13}$ Of the 274 SOF/VEL treated patients, the prevalence of NS5A RAS was $16 \%$. In patients with or without RAS, SVR was $88 \%$ (38/43) and $97 \%$ (225/231), respectively. Moreover, the SVR in patients with Y93H was 84\% (21/25). In Korea, the RAS test for GT3 patients is not available. Combination of ribavirin with SOF/VEL is recommended for TE CHC and TN- and TE-LC patients.

In a phase 2 study examining G/P treatment for 12 or 16 wk in TE patients without liver cirrhosis and for 16 wk in TE patients with liver cirrhosis (54\% PR, 46\% SOF+R with or without PEG-IFN), SVR were $91 \%$ (20/22), 96\% (21/22) and 96\% (45/47), respectively. ${ }^{11}$ 
Different from the 2017 KASL guidelines, the AASLD guidelines recommend SOF/VEL/VOX 12 wk for Y93-positive TN LC patients and Y93-positive TE CHC- or LC-patients. In a phase 3 study comparing SOF/VEL/VOX treatment for 8 wk with SOF/VEL for $12 \mathrm{wk}$ in GT 1-6 patients (for GT3, 77\% of the patients were TN with no liver cirrhosis), the SVR of GT3 patients was 99\% (91/92) and 97\% (86/89), respectively. ${ }^{14}$ In a phase 3 study comparing SOF/VEL/VOX treatment for 8 wk and SOF/VEL for 12 wk in genotype 3 patients with liver cirrhosis (IFN experienced 31\%), the SVR was $96 \%$ $(106 / 110)$ and $96 \%(105 / 109)$, respectively. ${ }^{14}$

\section{Treatment of TN and TE GT4 patients with CHC and CC}

The following six regimens are recommended for GT4 patients. LED+SOF treatment for $12 w k$ for TN patients and $12 w k+R$ or 24 wk for TE patients, EBR/GZP for 12 wk for TN patients and for those with previous IFN-based treatment relapser and 16wk+R for patients with previous on-treatment failure (including failure to suppress and breakthrough), OPr+R for $12 \mathrm{wk}, \mathrm{DCV}+\mathrm{SOF}$ for $12 \mathrm{wk}$ for patients without cirrhosis and $24 \mathrm{wk}$ or $12 \mathrm{wk}+\mathrm{R}$ for patients with cirrhosis. $\mathrm{G} / \mathrm{P}$ treatment for $8 \mathrm{wk}$ for patients without cirrhosis and for $12 \mathrm{wk}$ for patients with cirrhosis or SOF/VEL for 12 wk is one of the other treatment options although they are not approved in Korea yet.

Different from the AASLD/IDSA guidelines, the KASL guidelines recommend LED+SOF treatment for $12 \mathrm{wk}+\mathrm{R}$ or $24 \mathrm{wk}$ in TE CHC and LC patients. One phase 2 clinical trial using LED+SOF for $12 \mathrm{wk}$ in $21 \mathrm{CHC}$ patients (7 LC, 8 TE) showed a SVR of 95\%. ${ }^{32}$ Another phase 2 trial with the same treatment in $44 \mathrm{CHC}$ patients $(10 \mathrm{LC}$, $22 \mathrm{TE})$ showed a SVR of $93 \% .{ }^{33}$ Although the evidence is not robust yet, addition of ribavirin to $12 \mathrm{wk}$ LED+SOF treatment or extension of treatment duration to $24 \mathrm{wk}$ is recommended in these patients to improve SVR.

Different from the AASLD/IDSA guidelines, DCV+SOF treatment is one of the options for GT4 patients according to the KASL guidelines. In a retrospective study on 47 genotype 4 CHC patients, DCV+ SOF treatment for 12 wk showed a SVR of 100\% (32/32). ${ }^{34}$ In another retrospective study including 176 GT4 CHC patients (TE 82\%, LC 76\%), DCV+SOF with or without ribavirin for 12 or 24 wk showed $90 \%$ of SVR in total. In patients with cirrhosis, the SVR of DCV+SOF therapy with or without ribavirin was $97 \%, 88 \%$ respectively. ${ }^{35}$

Currently released HCV guidance in USA recommended LED/SOF treatment 12 wk for TN-CHC \& - LC, TE CHC patient and $12 w k+R$ in TE $L C$ patients. DCV+SOF is not recommended in GT4 patients. According to EASL 2016 guidelines, SIM+SOF are recommended in GT4 patients.

\section{Treatment of TN and TE GT5 and GT6 patients with $\mathrm{CHC}$ and $\mathrm{CC}$}

There are five available regimens for the treatment of GT5 and GT6 patients. LED+SOF treatment for 12 wk in TN and for $12 w k+R$ or 24 wk in TE patients, or DCV+SOF for 12 wk in TN and $12 w k+R$ or 24 wk in TE patients. G/P treatment for 8 wk in patients without cirrhosis and 12 wk for patients with cirrhosis or SOF/VEL for 12 wk is one of the other treatment options although they are not approved in Korea yet. PR for 24 wk may be considered if there is no available DAA treatment option for TN patients (i.e. CKD).

Different from the AASLD guidelines, LED+SOF treatment for 12 $w k+R$ or 24 wk is recommended for TE-GT5 and -GT6 patients in KASL guidelines. A phase 2 clinical trial using LED/SOF for 12 wk in 41 GT5 patients (9 LC, $20 \mathrm{TE})$ showed a SVR of 95\% (39/41) $)^{36}$ and another study with the same regimen in 25 GT6 patients (2 LC, 2 TE) showed a SVR of $96 \%(24 / 25) .{ }^{37}$ Different from the AASLD guidelines, DCV+SOF is a one of the treatment option for GT5 and GT6 patients in KASL guidelines. In a retrospective study, DCV+SOF treatment for 12 or 24 wk showed a SVR of $100 \%$ for both GT5 (25/25) and GT6 (5/5) patients. ${ }^{35}$

According to the AASLD/IDSA HCV guidance, LED/SOF treatment for 12 wk is recommended for TN and TE patients, whereas DCV+SOF is not recommended for GT5 and GT6 patients.

\section{TREATMENT APPROACH TO PATIENTS WITH DECOMPENSATED LIVER CIRRHOSIS (DC)}

The following three regimens are recommended for the treatment of GT1, GT4, GT5 and GT6 decompensated LC patients: LED+SOF for $12 \mathrm{wk}+\mathrm{R}$ (starting from $600 \mathrm{mg}$ ) or $24 \mathrm{wk}, \mathrm{DCV}+\mathrm{SOF}$ for $12 \mathrm{wk}+\mathrm{R}$ (starting from $600 \mathrm{mg}$ ) or $24 \mathrm{wk}$. VEL/SOF for $12 \mathrm{wk}+\mathrm{R}$ (weight based ribavirin) or $24 \mathrm{wk}$ is one of the other option although it is not approved in Korea yet.

There are two available regimens for the treatment of GT2 and GT3 DC patients: DCV+SOF for $12 \mathrm{wk}+\mathrm{R}$ (starting from $600 \mathrm{mg}$ ) or $24 \mathrm{wk}$ and VEL/SOF for $12 \mathrm{wk}+\mathrm{R}$ (weight based ribavirin) or $24 \mathrm{wk}$ although they are not approved in Korea yet.

In a multicenter randomized controlled trial of 108 patients with HCV genotypes 1 and 4 with DC (CTP class B or C, CTP scores $\leq 12$ ), patients were randomly assigned to receive a daily fixed-dose combination of LED/SOF with ribavirin (initial dose of $600 \mathrm{mg}$, increased as tolerated) for 12 or $24 \mathrm{wk}$. SVR of $12 \mathrm{wk}$ or $24 \mathrm{wk}$ in patients with CTP class B was $87 \%$ and $89 \%$. In CTP class C patients, SVR of 12 
wk or 24 wk was $86 \%$ and $87 \%$. $^{38}$

In a phase 3 trial, DCV+SOF+R treatment (initial low dose of 600 $\mathrm{mg}$ ) for 12 wk to sixty decompensated LC patients (HCV GT $1: 3$ : $2 / 4 / 6=45: 6: 9)$, resulted in an overall SVR of $83 \%$. SVR were $76 \%$ and $100 \%$ among patients with HCV GT1a and $1 \mathrm{~b}$, respectively. In patients with HCV GT1, SVR were $92 \%$ and $50 \%$ for patients with CTP class B and C, respectively. Among subjects with HCV GT3 and GT2/4/6, SVR 12 rates were $83 \%$ and $89 \%$, respectively. ${ }^{39}$

In a multicenter, open-label trial that included 267 previously treated and untreated patients with CTP class B (GT1a/GT1b/GT2/ GT3/GT4/GT6, 159/48/12/39/8/1), participants were randomly assigned to receive SOF/VEL treatment for $12 \mathrm{wk}, 12 \mathrm{wk}+\mathrm{R}$, or $24 \mathrm{wk}$. SVR was $88 \%, 94 \%$ and $93 \%$ in patients with GT1a, and $89 \%, 100 \%$ and $88 \%$ in patients with GT1b, respectively. For patients with GT2, the corresponding SVR were $100 \%, 100 \%$ and $75 \%$, while for patients with GT3, SVR were 50\%, 85\%, 50\%, respectively. Among patients with HCV GT4, all (100\%) achieved SVR to SOF/VEL-based regimens. ${ }^{40}$

In AASLD/IDSA guidance, DCV+SOF is only recommended for GT1 or GT4 patients.

\section{TREATMENT APPROACH FOR PATIENTS WITH LIVER TRANSPLANTATION (LT) OR OTHER EXTRA-HEPATIC ORGAN TRANSPLANTATION}

The following four regimens are recommended for the treatment of GT1 post LT patients: LED+SOF for 12 wk+R or 24 wk, DCV+SOF for 12 wk+R (starting from $600 \mathrm{mg}$ ) or $24 \mathrm{wk}$, G/P for 12 wk (if compensated) and OPrD for $24 w k+R$ (if F0-F2). The following three regimens are recommended for the treatment of GT2 post LT patients: DCV+SOF for 12 wk+R (starting from $600 \mathrm{mg}$ ) or 24 wk, G/P for $12 \mathrm{wk}$ (if compensated) and SOF+R for $24 \mathrm{wk}$. The following two regimens are recommended for the treatment of GT3 post LT patients: DCV+SOF for 12 wk+R (starting from $600 \mathrm{mg}$ ) or $24 \mathrm{wk}$ and $\mathrm{G} / \mathrm{P}$ for $12 \mathrm{wk}$ (if compensated). The following three regimens are recommended for the treatment of GT4, GT5 and GT6 post LT patients: LED+SOF for $12 w k+R$ or $24 w k, D C V+S O F$ for $12 w k+R$ (starting from $600 \mathrm{mg}$ ) or $24 \mathrm{wk}$ and G/P for 12 wk (if compensated).

In a multicenter, randomized controlled trial, patients treated with $\mathrm{LED} / \mathrm{SOF}+\mathrm{R}$ for either 12 or 24 wk, including GT1 or GT4 LT recipients $(n=229)$, SVR in stages F0 to F3 were $96 \%$ and $98 \%$ in the 12- and 24-wk arms, respectively. Patients with compensated cirrhosis showed a SVR of $96 \%$ in both the 12- and 24-wk. SVR was lower in patients with CTP class B LC (SVR $85 \%$ vs. $88 \%$ in the 12 - and 24-wk arms) or CTP class C cirrhosis (60\% vs. $75 \%$ in the 12 and 24-wk arms). ${ }^{38}$ In the open-label DCV+SOF+R (initial dose, 600 $\mathrm{mg}$ ) for $12 \mathrm{wk}$ in patients with recurrent HCV infection post-transplantation ( $n=53$, HCV GT 1:3:6=41:11:1), the overall SVR was 94\%. In GT1, GT3, and GT6, SVR were 95\% (39/41), 91\% (10/11), and $100 \%(1 / 1)$ respectively. ${ }^{39}$

The safety and efficacy of the $12 \mathrm{wk}$ G/P treatment was investigated in 100 patients (GT 1/2/3/4-6, 57\%/13\%/24\%/6\%) who developed recurrent HCV infection (F0-1 80\%, F2 6\%, and F3 14\%) after $\mathrm{LT}(\mathrm{n}=80)$ and renal transplantation $(\mathrm{n}=20)$. The overall SVR was $98 \%$ after 12 wk of $\mathrm{G} / \mathrm{P}$ therapy. ${ }^{41}$

A study, involving 34 LT recipients (29 GT1a) with no fibrosis or with mild fibrosis (Metavir fibrosis stage F0-F2) of GT1 patients, examined the use of fixed-dose combination OPr plus twice-daily dose of dasabuvir and weight-based ribavirin for $24 \mathrm{wk}$ and reported a SVR of $97 \%(33 / 34))^{42}$

In a pooled analysis of 10 studies with 333 patients with renal transplantation (GT1 88\%, TN 63\%, LC 25\%) receiving 12-24 wk of DAA therapy, SOF-based regimens were the most frequently used DAAs for recurrent hepatitis $C$ after renal transplantation. The overall SVR in post-renal transplant patients treated with DAA was $94.2 \%$. SVR was 67\% (10/15) in combination with SOF+R, 75\% (3/4) in combination with DCV+SOF, and $98 \%(158 / 161)$ in combination with LED/SOF with or without ribavirin. ${ }^{43}$

According to the AASLD treatment guidelines, for the treatment of GT1, GT4, GT5 and GT6 patients, LED+SOF+R treatment for 12 wk or G/P for 12 wk for non-LC patients, LED+SOF+R for 12 wk for CC patients can be considered. Alternatively, $\mathrm{DCV}+\mathrm{SOF}+\mathrm{R}$ (initial dose, $600 \mathrm{mg}$ ) for 12 wk, or SIM+SOF \pm R for 12 wk (for GT1, 4 only) or G/P for 12 wk in patient without LC or with CC can be considered. For DC with GT1, 4, 5, 6, LED/SOF+R (initial dose, $600 \mathrm{mg}$ ) for $12 \mathrm{wk}$ can be considered. For non-cirrhotic GT2 and GT3 patients, G/P for $12 \mathrm{wk}$, or DCV+SOF+R (initial dose, $600 \mathrm{mg}$ ) for 12 wk can be considered; For GT2 or GT3 patients with compensated cirrhosis, $\mathrm{DCV}+\mathrm{SOF}+\mathrm{R}$ (initial dose, $600 \mathrm{mg}$ ) for 12 wk or alternatively G/P for 12 wk, or SOF/VEL+R for 12 wk can be considered. In decompensated cirrhosis GT2 or GT3 patients, DCV+SOF+R (initial dose, $600 \mathrm{mg}$ ) for 12 wk or SOF/VEL+R for 12 wk can be considered.

\section{TREATMENT APPROACH TO PATIENTS WITH CHRONIC KIDNEY DISEASE (CKD)}

The following three regimens are recommended for GT1 patients with estimated GFR (eGFR) less than $30 \mathrm{~mL} / \mathrm{min}$ : EBR/GZR, OPrD (if 
GT1a, add ribavirin $200 \mathrm{mg} / \mathrm{d}$ ) and G/P. In GT1b patients, DCV+ASP for 24 wk may be considered. For GT2, GT3, GT4, GT5, GT6 patients with eGFR less than $30 \mathrm{~mL} / \mathrm{min}, \mathrm{G} / \mathrm{P}$ can be recommended. For GT4 patients with eGFR less than $30 \mathrm{~mL} / \mathrm{min}$, EBR/GRZ or OPrD+ ribavirin $200 \mathrm{mg} / \mathrm{d}$ can be considered. For GT2, GT3, GT5, GT6 patients with eGFR less than $30 \mathrm{~mL} / \mathrm{min}$ with no indication for DAA, $P R$ at reduced dose may be considered. In patients undergoing dialysis, PEG-IFN without ribavirin may be considered.

In genotype $1 \mathrm{HCV}$-infected patients with eGFR less than 30 $\mathrm{mL} / \mathrm{min}(\mathrm{n}=20,1 \mathrm{a} 65 \%)$ regardless of dialysis, $\mathrm{OPr}+\mathrm{D}$ with ribavirin (200 mg/day, GT1a) or without ribavirin (GT1b) for 12 wk resulted in SVR of 90\% (18/20) without dose modification. ${ }^{44}$ In GT1 HCV-infected patients with eGFR less than $30 \mathrm{~mL} / \mathrm{min}(\mathrm{n}=235,1 \mathrm{~b} 48 \%$, TN $80 \%$, LC 6\%) regardless of dialysis, EBR/GZR for 12 wk resulted in SVR of $99 \%$ without dose modification. ${ }^{45}$ In patients with GT1-6 HCV infection and renal impairment of variable degree, $G / P$ for 8-12 wk without dose modification resulted in SVR rates of $98 \%{ }^{46}$ In a retrospective Japanese study, DCV+ASV for 24 wk in dialysistreated GT1b patients resulted in SVR of 100\% without dose modification, and there was no significant adverse event. ${ }^{47}$ The dose for ASV should be reduced and prescribed at $100 \mathrm{mg}$ daily in nondialysis patients with eGFR less than $30 \mathrm{~mL} / \mathrm{min}$. Dose adjustment of PEG-IFN- $\alpha$ and ribavirin is required depending on the severity of kidney disease, because clearance is reduced according to the degree of impaired kidney function. The recommended treatment for patients with severe renal impairment (eGFR of $15-59 \mathrm{~mL} / \mathrm{min}$ ) is $135 \mu \mathrm{g}$ of PEG-IFN- $\alpha$-2a or $1 \mu \mathrm{g} / \mathrm{kg}$ of PEG-IFN- $\alpha$-2b together with $200-800 \mathrm{mg} /$ day of ribavirin twice per day with a gradual increase in dose. Patients on dialysis may be treated with either interferon alpha or PEG-IFN- $\alpha$; however, combination with ribavirin is not recommended.

According to AASLD/IDSA, EBR/GZR treatment for $12 \mathrm{wk}$ is recommended for GT1 and GT4 patients. G/P treatment for 8-16 wk is recommended for GT1, GT2, GT3, GT4, GT5 and GT6 patients with severe (eGFR less than $30 \mathrm{~mL} / \mathrm{min}$ ) or end stage CKD without dose adjustment.

\section{TREATMENT APPROACH FOR PATIENTS WHO FAILED PREVIOUS DAA TREATMENT}

For patients who failed previous DAA treatment, recommended regimens depends on previous treatment experience. SOF/VEL/VOX and $\mathrm{G} / \mathrm{P}$ are currently unavailable in Korea although some drugs may be prescribable in the future.
For patients who failed previous NS5A containing DAA, following 4 options are recommended: SOF/VEL/VOX 12 wk for GT1-6, G/P 16 wk for GT1 (G/P is recommended in patients who have been treated with regimens containing NS5A or NS3/4A PI, but not both), alternatively SOF+EBR/GZR 12 wk+R for GT1 or SOF+OPrD for GT1 (GT1a, $12 w k+R$ for $C H, 24 w k+R$ for LC; GT1b, 12 wk).

For patients who failed previous non-NS5A containing DAA, following 3 options are recommended: SOF/VEL/VOX 12 wk for GT14, G/P 12 wk for GT1 (G/P is recommended in patients who have been treated with regimens containing NS5A or NS3/4A PI, but not both). In GT1b or GT2, SOF/VEL treatment for 12 wk is an alternative treatment option.

For patients who failed previous SOF-containing DAA, following 5 options are recommended: SOF/VEL/VOX 12 wk for GT1-4, G/P 12 wk for GT1, 2, 4, 5, 6 and 16 wk for GT3. For GT1 patients, LED/ SOF (12 wk+R for $\mathrm{CH}$ and $24 w k+R$ for LC) can be considered. DCV+SOF 24 wk+R for GT2 and GT3 patients, SOF+EBR/GZR 12 wk for GT3 is one of the alternative options although the evidence is not strong.

The SVR was $70 \%$ after 12 wk re-treatment with LED/SOF in 54 patients with genotype $1 \mathrm{~b} C H C$ who did not respond to DCV+ASP treatment for $24 \mathrm{wk} .^{48}$ Twenty-five genotype $1 \mathrm{HCV}$ infected patients (22 GT1a, 3 GT1b, and 5 with cirrhosis) who failed the short-term combination therapy with SOF+EBR/GZR for 4,6 or 8 wk were retreated with SOF+EBR/GZR and ribavirin for 12 wk. The overall SVR rate was 100\% (25/25) and all patients with baseline NS3 RAS (17 patients) and NS5A RAS (14 patients) achieved SVR. ${ }^{49}$ Twenty-two GT1 infected HCV patients (20 GT 1a, 2 GT1b, 6 LC) who failed the previous DAA treatment (14 OPrD, 2 OPr) were treated with SOF+ OPrD with or without ribavirin. The overall SVR was 95\% (21/22). In GT1a patients, SVR of SOF+OPrD+R treatment for 12 wk in nonLC patients and for 24 wk in LC patients was 92\% (13/14) and 100\% (7/7), respectively. In GT1b patients, SVR of SOF+ OPrD treatment for 12 wks was $100 \%$ (2/2). All 18 patients with baseline RAS achieved SVR. ${ }^{50}$

A total of 263 patients with HCV infection (101 GT1a, 45 GT 1b, 4 other GT1, 5 GT 2, 78 GT3, 22 GT4, 1 GT5, 6 GT6, 1 unknown, 121 with $\mathrm{LC}$ ) who failed in previous DAA treatments including the NS5A inhibitor (161 NS5A and NS5B inhibitor, 83 NS5A and NS3 inhibitor, 18 NS5A inhibitor) were treated with SOF/VEL/VOX for 12 wk. ${ }^{51}$ The overall SVR was $96 \%$ (253/263). SVR of genotype 1a and 1b were $96 \%(97 / 101)$ and 100\% (45/45), respectively. The SVR of genotype 2, 3, 4, 5, and 6 was 100\% (5/5), 96\% (74/78), 91\% (20/22), 100\% (1/1), and 100\% (6/6), respectively. The SVR of patients without baseline RAS was $98 \%$ (42/43), and the SVR of pa- 
tients with RAS was 97\% (199/205). The SVR of 121 patients with cirrhosis was $93 \%(113 / 121){ }^{51}$

In a total, 333 patients with HCV infection (98 GT1a, 46 GT1b, 64 GT2, 106 GT3, 19 GT4, 153 with LC) who failed in previous nonNS5A DAA treatments (243 NS5B inhibitors, 84 NS5B+NS3 inhibitors, 5 NS3 inhibitors) were treated with either SOF/VEL/VOX or SOF/ VEL for 12 wk. $^{51}$ The overall SVR was $98 \%$ (178/182) and 90\% (136/ 151) respectively. The SVR for each genotype for SOF/VEL/VOX or SOF/VEL treatment were as follows; GT1a (98\% vs. $89 \%$ ), GT1b (96\% vs. $95 \%)$, GT2 (100\% vs. $97 \%$ ) and GT3 (96\% vs. $85 \%$ ). All 19 patients with genotype 4 who were treated with SOF/VEL/VOX for 12 wk achieved SVR (100\%). All 83 patients with baseline NS3 or NS5A RAS who were treated with SOF/VEL/VOX achieved SVR. The SVR of patients treated with SOF/VEL without or with baseline RAS was 89\% (67/75), 90\% (63/70), respectively. ${ }^{51}$

A total of 50 GT1 HCV infected patients without cirrhosis (42 GT1a, 8 GT1b) who failed previous DAA treatments (25 NS3 inhibitors, 8 NS5A inhibitors, 17 NS3 inhibitors and NS5A inhibitors), were treated with $\mathrm{G} / \mathrm{P}$ with or without ribavirin for $12 \mathrm{wk}$. The overall SVR was $92 \%(46 / 50)$. $^{52}$ Ninety-one patients with CHC (67 GT1a, 18 GT1b, 2 other GT1, 4 GT4, 27 LC) who had failed previous DAA treatments (34 NS5A inhibitors, 27 NS3 inhibitors, 30 NS3 and NS5A inhibitors), were treated with G/P for 12 or 16 wk. Overall, the SVR was $89 \%$ (39/44) and $91 \%$ (43/47), respectively. ${ }^{53}$ In patients who failed previous NS3 inhibitor treatment, SVR for 12 or $16 \mathrm{wk}$ of treatment were $100 \%$ (14/14) and 100\% (13/13). In patients who failed previous NS5A inhibitor treatment, SVR of each treatment duration was $88 \%(14 / 16)$ and $94 \%$ (17/18). In patients who failed previous NS3 and NS5A inhibitor treatment, SVR of 12- or -16 wk of G/P treatment is $79 \%(11 / 14)$ and $81 \%(13 / 16)$. In patients without baseline RAS, SVR of 12 and 16 wk of G/P was 100\% (13/13) and $100 \%$ (13/13), respectively. In patients with NS3 RAS, SVR was $100 \%(2 / 2)$ and $100 \%(4 / 4)$. In patients with NS5A RAS, SVR was $83 \%$ (20/24) and $96 \%$ (22/23). In patients who have both of NS3 and NS5A RAS, SVR was $80 \%(4 / 5)$ and $25 \%(1 / 4)$, respectively. ${ }^{53}$ Therefore, based on the clinical results so far, G/P seems to have a limited efficacy in patients who have both of NS3 RAS and NS5A RAS.

The SVR of 12 wk of treatment with LED/SOF 24 wk was $100 \%$ in 14 patients with GT1 CHC (8 GT1a, 6 GT1b) who failed the SOF+R treatment for 24 wk. ${ }^{54}$ Fifty-one patients (30 GT1a, 20 GT1b, 1 GT3a, 14 with LC) who failed previous HCV therapy (25 SOF+PR, $20 \mathrm{SOF}+\mathrm{R}$, 6 PR) were treated with LED/SOF+R for $12 w k$. The SVR was $98 \%$ (50/51). ${ }^{55} \mathrm{~A}$ total of 52 patients with CHC (44 GT1, 2 GT2, 4 GT3, 3 GT4) who had previous treatment experience were treated with
DCV+SOF for 12 wk. The SVR was $98 \%$ (51/52), however the number of patients with GT2 and GT3 was very limited..$^{56}$ In a study of genotype 3 patients treated with DCV+SOF for 12 wk, patients with prior treatment experience with SOF and ribavirin combination or SOF+PR did not show satisfactory SVR $(71 \%, 5 / 7) .{ }^{30}$ Based on these limited SVR of $12 \mathrm{wk}$ combination, DCV+SOF+R for $24 \mathrm{wk}$ may be an alternative option.

In a genotype $3 \mathrm{CHC}$ patient with cirrhosis, 53 patients who failed previous treatment (including $2 \mathrm{SOF}+\mathrm{R}$ therapy) were treated with EBR/GZR and SOF for $12 w k$, or with EBR/GZR+SOF with ribavirin for 12 wk, or with EBR/GZR+SOF with ribavirin for 16 wk. The SVR were 100\% (17/17), 94\% (17/18) and 94\% (17/18), respectively. ${ }^{57}$

In GT 1, 2, 4, 5, and 6 CHC patients without cirrhosis, patients were treated with G/P for 8 or 12 wk. ${ }^{58,59}$ The SVR was $97-99 \%$ and $99-100 \%$ for 8 and 12 wk of treatment, respectively. A total of 146 patients with genotype 1, 2, 4, 5, and 6 CHC patients with cirrhosis (including 36 TE patients and 11 patients who had SOFbased treatment) were treated with G/P for 12 wk and the SVR was $99 \% .^{10} \mathrm{~A}$ total of 131 patients with genotype $3 \mathrm{CHC}$ were treated with G/P for 12 or 16 wk. Among them, 91 TE patients (including 42 patients with SOF-based treatment) were included. SVRs of $12 \mathrm{wk}$ treatment in patients without cirrhosis, 16 wk treatment in patients without cirrhosis, and 16 wk treatment in patients with cirrhosis were $91 \%$ (20/22), 96\% (21/22) and 96\% (45/47), respectively.

According to the currently released AASLD/IDSA HCV guidance, treatment recommendations are follows as; for non-LC or LC GT1 patients who failed NS5A-containing regimen, SOF/VEL/VOX 12 wk or G/P 16wk (except for the PI-containing DAA regimen). For non-LC GT1 patients who failed non-NS5A or SOF-containing regimen, SOF/VEL/VOX 12 wk or G/P 12 wk for GT1a patients and G/P 12 wk or SOF/VEL 12 wk for GT1b patients, or LED/SOF+R 12 wk can be considered. For LC GT1 patients who failed non-NS5A or SOF-containing regimen, SOF/VEL/VOX 12 wk or G/P 12 wk for GT1a patients and G/P 12 wk or SOF/VEL 12 wk for GT1b patients can be considered. For GT2 with SOF experienced patients, SOF/VEL 12 wk or G/P 12 wk is recommended. For GT3 DAA experienced patients, SOF/VEL/VOX treatment 12 wk and 12 wk+R for LC with NS5A failure is recommended. For GT4, 5, 6 who failed previous DAA treatment, SOF/VEL/VOX 12wk is recommended.

\section{Conflicts of Interest}

Received grants from Pharmaking, Bayer, BMS, Dong-A, Green Cross lab cell. 


\section{REFERENCES}

1. KASL clinical practice guidelines: management of hepatitis C. Clin Mol Hepatol 2014;20:89-136.

2. KASL clinical practice guidelines: management of hepatitis C. Clin Mol Hepatol 2016;22:76-139.

3. Korea Association for the Study of the Liver. KASL Clinical Practice Guidelines, Management of hepatitis C: Treatment of chronic hepatitis C. KASL web site, <http://www.kasl.org/bbs/index.html?code=guide\& category $=$ \&gubun $=\& i d x=\&$ page $=1$ \&number $=3359 \&$ mode $=v i e w \& 0$ rder $=\&$ sort=\&keyfield $=\& k e y=>$. Accessed 1 Nov 2017.

4. American Association for the Study of Liver Diseases and the Infectious Diseases Society of America (AASLD/IDSA). HCV Guidance: Recommendations for Testing, Managing, and Treating Hepatitis C. AASLD web site, <http://www.hcvguidelines.org>. Accessed 1 Apr 2017.

5. EASL Recommendations on Treatment of Hepatitis C 2016. J Hepatol 2017;66:153-194.

6. Zeuzem S, Ghalib R, Reddy KR, Pockros PJ, Ben Ari Z, Zhao Y, et al. Grazoprevir-elbasvir combination therapy for treatment-Naive cirrhotic and noncirrhotic patients with chronic hepatitis $C$ virus genotype 1, 4, or 6 infection: a randomized trial. Ann Intern Med 2015;163:113.

7. Jacobson IM, Asante-Appiah E, Wong P, Howe A, Wahl J, Robertson $M$, et al. Prevalence and impact of baseline NS5A resistance associated variants (RAVs) on the efficacy of elbasvir/grazoprevir (EBR/ GZR) against GT1a infection [Abstract]. Hepatology 2015;62:1393A1394A.

8. Zeuzem S, Feld J, Wang S. ENDURANCE-1: Efficacy and Safety of 8versus 12-week Treatment with ABT-493/ABT-530 in patients with Chronic HCV Genotype 1 Infection [Abstract]. Hepatology 2016;64:132A-133A.

9. Pol S, Pockros PJ, Pugatch D, Brau N, Landis C, Elkhashab M, et al. Safety and efficacy of glecaprevir/pibrentasvir in adults with chronic hepatitis C virus infection genotype 1-6 and chronic kidney disease: an integrated analysis [Abstract]. Gastroenterology 2017;152:S1062S1063.

10. Forns X, Lee SS, Valdes J, Lens S, Ghalib R, Aguilar H, et al. Glecaprevir plus pibrentasvir for chronic hepatitis $C$ virus genotype $1,2,4,5$, or 6 infection in adults with compensated cirrhosis (EXPEDITION-1): a single-arm, open-label, multicentre phase 3 trial. Lancet Infect Dis 2017; 17:1062-1068.

11. Wyles D, Poordad F, Wang S, Alric L, Felizarta F, Kwo PY, et al. Glecaprevir/pibrentasvir for hepatitis $C$ virus genotype 3 patients with cirrhosis and/or prior treatment experience: a partially randomized phase 3 clinical trial. Hepatology 2018;67:514-523.

12. Feld JJ, Jacobson IM, Hézode C, Asselah T, Ruane PJ, Gruener N, et al. Sofosbuvir and Velpatasvir for HCV Genotype 1, 2, 4, 5, and 6 Infection. N Engl J Med 2015;373:2599-2607.

13. Foster GR, Afdhal N, Roberts SK, Bräu N, Gane EJ, Pianko S, et al.
Sofosbuvir and velpatasvir for HCV genotype 2 and 3 infection. N Engl J Med 2015;373:2608-2617.

14. Jacobson IM, Lawitz E, Gane EJ, Willems BE, Ruane PJ, Nahass RG, et al. Efficacy of 8 weeks of sofosbuvir, velpatasvir, and voxilaprevir in patients with chronic HCV infection: 2 phase 3 randomized trials. Gastroenterology 2017;153:113-122.

15. Guyatt GH, Oxman AD, Vist GE, Kunz R, Falck-Ytter Y, Alonso-Coello P, et al. GRADE: an emerging consensus on rating quality of evidence and strength of recommendations. BMJ 2008;336:924-926.

16. Kao JH, Lee YJ, Heo J, Ahn SH, Lim YS, Peng CY, et al. All-oral daclatasvir plus asunaprevir for chronic hepatitis $\mathrm{C}$ virus (HCV) genotype 1b infection: a sub-analysis in Asian patients from the HALLMARK DUAL study. Liver Int 2016;36:1433-1441.

17. McPhee F, Suzuki Y, Toyota J, Karino Y, Chayama K, Kawakami Y, et al. High sustained virologic response to daclatasvir plus asunaprevir in elderly and cirrhotic patients with hepatitis C virus genotype $1 \mathrm{~b}$ without baseline NS5A polymorphisms. Adv Ther 2015;32:637-649.

18. Backus LI, Belperio PS, Shahoumian TA, Loomis TP, Mole LA. Realworld effectiveness of ledipasvir/sofosbuvir in 4,365 treatment-naive, genotype 1 hepatitis C-infected patients. Hepatology 2016;64:405414.

19. Kowdley KV, Sundaram V, Jeon CY, Qureshi K, Latt NL, Sahota A, et al. Eight weeks of ledipasvir/sofosbuvir is effective for selected patients with genotype 1 hepatitis C virus infection. Hepatology 2017;65:1094-1103.

20. Pol S, Bourliere M, Lucier S, Hezode C, Dorival C, Larrey D, et al. Safety and efficacy of daclatasvir-sofosbuvir in HCV genotype 1-monoinfected patients. J Hepatol 2017;66:39-47.

21. Afdhal N, Reddy KR, Nelson DR, Lawitz E, Gordon SC, Schiff E, et al. Ledipasvir and sofosbuvir for previously treated HCV genotype 1 infection. N Engl J Med 2014;370:1483-1493.

22. Zeuzem S, Mizokami M, Pianko S, Mangia A, Han KH, Martin R, et al. NS5A resistance-associated substitutions in patients with genotype 1 hepatitis C virus: prevalence and effect on treatment outcome. J Hepatol 2017;66:910-918.

23. Lawitz E, Mangia A, Wyles D, Rodriguez-Torres M, Hassanein T, Gordon SC, et al. Sofosbuvir for previously untreated chronic hepatitis $C$ infection. N Engl J Med 2013;368:1878-1887.

24. Jacobson IM, Gordon SC, Kowdley KV, Yoshida EM, Rodriguez-Torres $M$, Sulkowski MS, et al. Sofosbuvir for hepatitis C genotype 2 or 3 in patients without treatment options. N Engl J Med 2013;368:18671877.

25. Welzel TM, Nelson DR, Morelli G, Di Bisceglie A, Reddy RK, Kuo A, et al. Effectiveness and safety of sofosbuvir plus ribavirin for the treatment of HCV genotype 2 infection: results of the real-world, clinical practice HCV-TARGET study. Gut 2017;66:1844-1852.

26. Mangia A, Susser S, Piazzolla V, Agostinacchio E, De Stefano G, Palm-

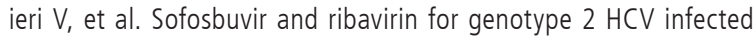


patients with cirrhosis: a real life experience. J Hepatol 2017;66:711717.

27. Lee SW, Lee HL, Han NI, Kim HY, Kim CW, You CR, et al. Real-life experience of sofosbuvir and ribavirin for genotype $2 \mathrm{HCV}$ infected Korean patients: a multicenter cohort study [Abstract]. Clin Mol Hepatol 2017; 23(Suppl 3):62-63.

28. Sulkowski MS, Gardiner DF, Rodriguez-Torres M, Reddy KR, Hassanein $T$, Jacobson I, et al. Daclatasvir plus sofosbuvir for previously treated or untreated chronic HCV infection. N Engl J Med 2014;370:211-221.

29. Ippolito AM, Milella M, Messina V, Conti F, Cozzolongo R, Morisco F, et al. HCV clearance after direct-acting antivials in patients with cirrhosis by stages of liver impairment: the ITAL-C network study. Dig Liver Dis 2017:49:1022-1028.

30. Nelson DR, Cooper JN, Lalezari JP, Lawitz E, Pockros PJ, Gitlin N, et al. All-oral 12-week treatment with daclatasvir plus sofosbuvir in patients with hepatitis C virus genotype 3 infection: ALLY-3 phase III study. Hepatology 2015;61:1127-1135.

31. Alonso S, Riveiro-Barciela M, Fernandez I, Rincón D, Real Y, Llerena S, et al. Effectiveness and safety of sofosbuvir-based regimens plus an NS5A inhibitor for patients with HCV genotype 3 infection and cirrhosis. Results of a multicenter real-life cohort. J Viral Hepat 2017;24:304311.

32. Kohli A, Kapoor R, Sims Z, Nelson A, Sidharthan S, Lam B, et al. Ledipasvir and sofosbuvir for hepatitis $C$ genotype 4: a proof-of-concept, single-centre, open-label phase 2a cohort study. Lancet Infect Dis 2015;15:1049-1054.

33. Abergel A, Metivier S, Samuel D, Jiang D, Kersey K, Pang PS, et al. Ledipasvir plus sofosbuvir for 12 weeks in patients with hepatitis $C$ genotype 4 infection. Hepatology 2016;64:1049-1056.

34. Fontaine H, Hezode C, Zoulim F, Samuel D, Bourliere M, Haour G, et al. Efficacy of the oral sofosbuvir-based combination in HCV genotype 4-mono-infected patients from the french observational cohort anrs CO22 hepather [Abstract]. J Hepatol 2015;62(Suppl 2):S278-S279.

35. Hézode C, Abergel A, Chas J, Conti F, Cotte L, Tateo M, et al. Response to daclatasvir and sofosbuvir, with or without ribavirin, among patients in the French daclatasvir ATU programme infected with HCV genotype 4, 5, and 6 [Abstract]. J Hepatol 2016;64:S755.

36. Abergel A, Asselah T, Metivier S, Kersey K, Jiang D, Mo H, et al. Ledipasvir-sofosbuvir in patients with hepatitis $C$ virus genotype 5 infection: an open-label, multicentre, single-arm, phase 2 study. Lancet Infect Dis 2016;16:459-464.

37. Gane EJ, Hyland RH, An D, Svarovskaia E, Pang PS, Brainard D, et al. Efficacy of ledipasvir and sofosbuvir, with or without ribavirin, for 12 weeks in patients with HCV genotype 3 or 6 infection. Gastroenterology 2015;149:1454-1461.

38. Charlton M, Everson GT, Flamm SL, Kumar P, Landis C, Brown RS Jr, et al. Ledipasvir and sofosbuvir plus ribavirin for treatment of HCV infection in patients with advanced liver disease. Gastroenterology
2015;149:649-659.

39. Poordad F, Schiff ER, Vierling JM, Landis C, Fontana RJ, Yang R, et al. Daclatasvir with sofosbuvir and ribavirin for hepatitis $C$ virus infection with advanced cirrhosis or post-liver transplantation recurrence. Hepatology 2016;63:1493-1505.

40. Curry MP, O'Leary JG, Bzowej N, Muir AJ, Korenblat KM, Fenkel JM, et al. Sofosbuvir and velpatasvir for HCV in patients with decompensated cirrhosis. N Engl J Med 2015;373:2618-2628.

41. Reau N, Kwo PY, Rhee S, Brown RS Jr, Agarwal K, Angus P, et al. MAGELLAN-2: safety and efficacy of glecaprevir/pibrentasvir in liver or renal transplantation adults with chronic hepatitis C genotype 1-6 infection [Abstract]. J Hepatol 2017;66(Suppl):S90-S91.

42. Kwo PY, Mantry PS, Coakley E, Te HS, Vargas HE, Brown R Jr, et al. An interferon-free antiviral regimen for HCV after liver transplantation. N Engl J Med 2014;371:2375-2382.

43. Cholongitas E, Pipili C, Papatheodoridis GV. Interferon-free regimens in patients with hepatitis C infection and renal dysfunction or kidney transplantation. World J Hepatol 2017;9:180-190.

44. Pockros PJ, Reddy KR, Mantry PS, Cohen E, Bennett M, Sulkowski MS, et al. Efficacy of direct-acting antiviral combination for patients with hepatitis C virus genotype 1 infection and severe renal impairment or end-stage renal disease. Gastroenterology 2016;150:15901598.

45. Roth D, Nelson DR, Bruchfeld A, Liapakis A, Silva M, Monsour H Jr, et al. Grazoprevir plus elbasvir in treatment-naive and treatmentexperienced patients with hepatitis C virus genotype 1 infection and stage 4-5 chronic kidney disease (the C-SURFER study): a combination phase 3 study. Lancet 2015;386:1537-1545.

46. Gane E, Lawitz E, Pugatch D, Papatheodoridis G, Bräu N, Brown A, et al. Glecaprevir and pibrentasvir in patients with HCV and severe renal impairment. N Engl J Med 2017;377:1448-1455.

47. Toyota J, Karino Y, Suzuki F, Ikeda F, Ido A, Tanaka K, et al. Daclatasvir/ asunaprevir/beclabuvir fixed-dose combination in Japanese patients with HCV genotype 1 infection. J Gastroenterol 2017;52:385-395.

48. Akuta N, Sezaki H, Suzuki F, Fujiyama S, Kawamura Y, Hosaka T, et al. Ledipasvir plus sofosbuvir as salvage therapy for HCV genotype 1 failures to prior NS5A inhibitors regimens. J Med Virol 2017;89:1248 1254.

49. Lawitz E, Poordad F, Gutierrez JA, Wells JT, Landaverde CE, Evans B, et al. Short-duration treatment with elbasvir/grazoprevir and sofosbuvir for hepatitis C: a randomized trial. Hepatology 2017;65:439-450.

50. Poordad F, Bennett M, Sepe T, Cohen E, Reindollar RW, Everson G, et al. Ombitasvir/paritaprevir/r, dasabuvir, and sofosbuvir treatment of patients with HCV genotype 1 infection who failed a prior course of DAA therapy: the quartz-I study. J Hepatol 2016;64(Suppl):S767-S768.

51. Bourlière M, Gordon SC, Flamm SL, Cooper CL, Ramji A, Tong M, et al. Sofosbuvir, velpatasvir, and voxilaprevir for previously treated HCV infection. N Engl J Med 2017;376:2134-2146. 
52. Poordad F, Felizarta F, Asatryan A, Sulkowski MS, Reindollar RW, Landis CS, et al. Glecaprevir and pibrentasvir for 12 weeks for hepatitis $C$ virus genotype 1 infection and prior direct-acting antiviral treatment. Hepatology 2017;66:389-397.

53. Poordad F, Pol S, Asatryan A, Buti M, Shaw DR, Hézode C, et al. MAGELLAN-1, part 2: glecaprevir and pibrentasvir for 12 or 16 weeks in patients with chronic HCV genotype 1 or 4 and prior direct-acting antiviral treatment failure [Abstract]. Gastroenterology 2017;66(Suppl 1):S1057.

54. Osinusi A, Kohli A, Marti MM, Nelson A, Zhang X, Meissner EG, et al. Re-treatment of chronic hepatitis $C$ virus genotype 1 infection after relapse: an open-label pilot study. Ann Intern Med 2014;161:634-638.

55. Wyles D, Pockros P, Morelli G, Younes Z, Svarovskaia E, Yang JC, et al. Ledipasvir-sofosbuvir plus ribavirin for patients with genotype 1 hepatitis $C$ virus previously treated in clinical trials of sofosbuvir regimens. Hepatology 2015;61:1793-1797.

56. Wyles DL, Ruane PJ, Sulkowski MS, Dieterich D, Luetkemeyer A, Mor- gan TR, et al. Daclatasvir plus sofosbuvir for HCV in patients coinfected with HIV-1. N Engl J Med 2015;373:714-725.

57. Foster GR, Agarwal K, Cramp ME, Moreea S, Barclay S, Collier J, et al. Elbasvir/grazoprevir plus sofosbuvir in treatment naive and treatment experienced cirrhotic patients with hepatitis $C$ virus genotype 3 infection treated for 8, 12, or 16 weeks: final results fothe C-ISLE study [Abstract]. J Hepatol 2017;66(Suppl):S503.

58. Kowdley KV, Colombo M, Zadeikis N, Mantry PS, Calinas F, Aguilar $\mathrm{HI}$, et al. ENDURANCE-2: safety and efficacy of ABT-493/ABT-530 in hepatitis $C$ virus genotype 2 -infected patients without cirrhosis: a randomized, double-blind, placebo-controleed study [Abstract]. Hepatology 2016;64(Suppl):39A.

59. Hassanein T, Wyles D, Wang S, Kwo PY, Shiffman ML, Younes Z, et al. SURVEYOR-II, Part 4: Glecaprevir/pibrentasvir demonstrates high SVR rates in patients with HCV genotype 2, 4, 5, or 6 infection without cirrhosis following an 8-weeks treatment duration [Abstract]. Hepatology 2016;64(Suppl):1128A. 